\title{
Acibenzolar-S-Methyl Inhibits MEK1/2 Signaling in SH-SY5Y Neuroblastoma Cells
}

\author{
Aysegul Yildiz' \\ 'Mugla Sitki Kocman University, Faculty of Science, Department of Molecular Biology and Genetics, Mugla, Turkey
}

ORCID IDs of the authors: A.Y. 0000-0001-6356-7459

Please cite this article as: Yildiz A. Acibenzolar-S-Methyl Inhibits MEK1/2 Signaling in SH-SY5Y Neuroblastoma Cells. Eur J Biol 2019; 78(2): 83-88. DOI: 10.26650/EurJBiol.2019.0020

\begin{abstract}
Objective: Targeted cancer therapy using targeted cell proliferation inhibitors has become increasingly more critical. Studies conducted over the last decade have shown that non-steroidal drugs containing salicylic acid (SA) such as aspirin reduce mortality in many cancers. From this perspective, there are data suggesting SA as a potential inhibitor of the mitogenic MEK1/2 (mitogen-activated-protein-kinase, MAPK), extracellular-signal regulated-protein-kinase (ERK)) signaling, which could be highly effective in the prevention of proliferation in cancer. To date, no study has been conducted on the effect of SA on MEK $1 / 2$ signaling in neuroblastoma cells. Thus, the aim of this study is to reveal whether SA has an effect on MEK1/2 signaling in neuroblastoma cancer which is a frequent pediatric cancer with poor prognosis.
\end{abstract}

Materials and Methods: The purpose of this study was to investigate whether a SA analog acibenzolar-S-methyl had an effect on the MEK1/2 signaling pathway and on cell viability in SH-SY5Y neuroblastoma cells by MTS (3-(4,5-dimethylthiazol2-yl)-5-(3-carboxymethoxyphenyl)-2-(4-sulfophenyl)-2H-tetrazolium) cell viability analysis and MEK1/2 and active caspase-3 detection by western blotting technique.

Results: MTS cell viability test indicated that $10 \mathrm{mM}$ acibenzolar-S-methyl reduces cell viability by $50 \%$. Western blotting results of $10 \mathrm{mM}$ acibenzolar-S-methyl-treated cells showed that MEK1/2 signaling was significantly inhibited in SH-SY5H cells. Besides, an increase in active-caspase-3 levels provided insight into acibenzolar-S-methyl's apoptotic effect which needs further morphological apoptotic data.

Conclusion: Our research is the first to show that SA analog acibenzolar-S-methyl negatively affects MEK1/2 signaling causing the death of SH-SY5Y neuroblastoma cells. Our results can give insight not only into understanding the mechanisms of carcinogenesis but also into developing effective treatment methods.

Keywords: Salicylic acid, Acibenzolar-S-Methyl, MEK1/2, SH-SY5Y, neuroblastoma cancer

\section{INTRODUCTION}

Signal transduction is an important part of a complex system which organizes many cellular activities in the cell and regulates their behavior. Errors in signal transduction are, therefore the main cause of diseases such as cancer, autoimmunity, and diabetes. Thus, metabolic functions related to signal transduction have become the most important focus of cancer biology (1).

In this regard, targeting mitogenic and/or survival signals in order to suppress cell proliferation or cell growth through targeted inhibitors is getting more attention. By means of targeted inhibitors, successful results can be achieved by suppressing cell proliferation or cell growth during tumorigenesis. To this end, several important studies have been performed and results showed that low dose aspirin which contains salicylic acid (SA) reduces the incidence and mortality of colorectal, breast, gastric, lung or other cancers (2-6).

Furthermore, researchers examining the effect of SA on a hepatoma cell line have shown that SA can inhibit proliferation and induce apoptosis of the hepatoma cell line in a time and dose-dependent manner (7). In addition, in a study of human colorectal tumor cell lines, 
the effect of salicylate, aspirin metabolite, on these cell lines was determined. Salicylate showed dose-dependent inhibitory effects in all cell lines (8). All these studies indicate that SA may be protective and preventive against cancer.

At this point, one could pose a question regarding what the mechanism of the apoptotic action of salicylate in cancer is. In this context, the high number of protein kinases modulated by salicylate may provide a strong explanation for the apoptotic effect of salicylate on cancer cells. From this perspective, a considerable amount of data suggests that SA may have an effect on the mitogenic MAPK/ERK (mitogen-activated protein kinase/extracellular signal-regulated kinases) signaling pathway, one of the cell's most vital signaling pathways $(9,10)$.

MAPK enzymes found in all eukaryotic cells are the intersections and/or junctions of mitogenic stimuli received by different receptors. In response to the stimuli it receives, the intracellular signal is transferred to the small oncogenic G-protein Ras and then to the Raf (MEK kinase) protein, which then activates MEK1/2 (MAPK/ERK kinase or MAP kinase kinase) signal proteins. Activated MEK1/2 phosphorylates and activates ERK1/2 which ultimately regulates essential cellular events such as gene expression, mitosis, cell viability, apoptosis, cellular metabolism, differentiation and motility (11). As is evident from its function, MEK $1 / 2$ is one of the key regulators of MAPK/ERK signaling cascade.

There is a study conducted with A549 human lung cancer cells demonstrating that SA may have a suppressing effect on this vital signaling pathway (9). In the afore-mentioned study the said effect was demonstrated by disrupting the MAPK signaling pathway by inhibiting the binding of c-Raf to the Ras protein (9). This data is very important given the role of the MAPK signaling pathway in processes such as cell proliferation, differentiation, and survival.

Although it is true that in many cancers inhibiting MEK1/2 signaling results in apoptotic death, in certain types of cancer such as melanoma, inhibiting MEK $1 / 2$ signaling could contrarily contribute to resistance to apoptosis (10) pointing out the heterogeneous nature of the molecular and genetic basis of cancer.

However, little is known about the role and effect of MEK1/2 signaling in neuroblastoma cancer, and no studies have as yet been conducted on the effect of SA on this signaling pathway. There are only a few studies indicating that the MAPK signaling pathway plays a role in the transformation of neuroblastoma cells into transformed phenotype and in gaining resistance to chemotherapy (12). In addition, we have demonstrated through our recently completed study that inhibiting MEK1/2 signaling by specific MEK1/2 inhibitor U0126 in SH-SY5Y neuroblastoma cells results in a significant decrease in cell viability (unpublished data).

Taking into account that neuroblastoma is the most common type of pediatric extracranial solid tumor in which $98 \%$ of the patients are under 10 years of age, and considering that the disease has a very poor prognosis with a $30 \%$ long-term survival rate $(13,14)$, it would be of great value to investigate whether SA has an effect on the MEK1/2 signaling and correlatively on cell viability in neuroblastoma cells. With this aim in mind, we treated $\mathrm{SH}-\mathrm{SY} 5 \mathrm{H}$ neuroblastoma cell line with an SA analog, acibenzolar-S-methyl and we analyzed the effect of acibenzolar-S-methyl on MEK $1 / 2$ signaling and on cell viability. Western blotting results of acibenzolar-S-methyl-treated cells showed that MEK1/2 signaling was significantly inhibited. In addition, we determined that active caspase-3 levels increased upon acibenzolar-S-methyl treatment. Although this result is promising for the apoptotic effect of acibenzolar-S-methyl in SH-SY5Y cells, it needs further morphological analysis to be able to exactly point out apoptosis in acibenzolar-S-methyltreated SH-SY5Y cells.

In this study, we showed for the first time that salicylic acid analog acibenzolar-S-methyl negatively affects MEK1/2 signaling causing death of SH-SY5Y neuroblastoma cells. Our study may become a guide not only for choosing the right targets but also for choosing the right weapons in the fight against cancer.

\section{MATERIALS AND METHODS}

\section{Cell Culture}

$\mathrm{SH}-\mathrm{SY} 5 \mathrm{Y}$ neuroblastoma cells were cultured at $37^{\circ} \mathrm{C}$ with $5 \% \mathrm{CO}_{2}$ at 1 atmospheric pressure in high-glucose Dulbecco's modified Eagle medium (DMEM) containing 10\% fetal bovine serum (FBS), $2 \mathrm{mM} \mathrm{L}$-Glutamine and antibiotics (100 U/mL penicillin and $100 \mu \mathrm{g} / \mathrm{mL}$ streptomycin). When the cells reached sufficient confluence, they were washed with sterile phosphate buffered saline (PBS). Then, the cells were removed from the petri dish with a $10 \mathrm{X}$ stock solution of $2.5 \%$ sterile trypsin-ethylene diamine tetraacetic acid (Trypsin-EDTA).

\section{Acibenzolar-S-methyl Treatment of SH-SY5Y cells}

Firstly, SH-SY5Y cells were grown on 96 well-plates as $10^{4}$ cells/well and they were incubated at $37^{\circ} \mathrm{C}$ with $\% 5 \mathrm{CO}_{2}$ for 18-20 hours in order to obtain $60-80 \%$ confluency. Then, Acibenzolar-S-methyl (Syngenta) was applied at 1, 10, 15 and $20 \mathrm{mM}$ concentrations on cultured cells in order to investigate the effect of different concentrations of acibenzolar-S-methyl on SH-SY5Y cells to be able to determine the half-maximal inhibitory concentration (IC50). For this aim, treated cells were incubated at $37^{\circ} \mathrm{C}$ with $\% 5 \mathrm{CO}_{2}$ for 24 and 48 hours.

\section{MTS Cell Viability Assay}

In order to investigate IC50 value for acibenzolar-S-methyl on SH-SY5Y cells, MTS Cell Viability Assay (ab197010, Abcam) was used according to the manufacturers' instructions. Briefly, $20 \mu \mathrm{l}$ MTS solution was added onto both cultured control SHSY5Y cells and SH-SY5Y cells which were treated with different concentrations of acibenzolar-S-methyl and incubated for 24 and 48 hours. Then the cells were incubated for 3.5 hours at $37^{\circ} \mathrm{C}$ with $5 \% \mathrm{CO}_{2}$. After that, spectrophotometric analysis was performed at $490 \mathrm{~nm}$ wavelength for absorbance measurement 
using SpectraMax ${ }^{\circledast}$ i3 Platform (Molecular Devices, LLC, San Jose, CA, USA). Cell viability data were obtained from three independent experiments and performed in triplicate wells.

\section{Western Blotting}

Total protein from acibenzolar-S-methyl-treated and control SH-SY5Y cells was extracted using Whole Cell Extraction Kit (2910, Millipore). The protein concentration was measured using the Qubit ${ }^{\mathrm{TM}}$ Protein Assay Kit (Thermo Fisher Scientific) and Qubit ${ }^{\mathrm{TM}}$ 3.0 Fluorometer. Equal amounts of samples (100 $\mu \mathrm{g}$ protein/ lane) were separated by SDS-PAGE (12.5\% sodium dodecyl sulfate-polyacrylamide electrophoresis according to the molecular weight of the proteins) and transferred to nitrocellulose membrane (sc-3724, Santa Cruz Biotechnology) using Trans-Blot Turbo Transfer System (BioRad). The membranes were blocked in $5 \% \mathrm{BSA} /$ tris buffered salinetween (TBS-T) for $1 \mathrm{~h}$ at room temperature, stained with the primary antibodies overnight at $4{ }^{\circ} \mathrm{C}$ and with the secondary antibody for $1 \mathrm{~h}$ at room temperature. The following primary antibodies were used at 1:1000 dilutions; mouse monoclonal anti- MEK1/2 (sc-81504, Santa Cruz Biotechnology), mouse monoclonal anti-calnexin (sc-80645, Santa Cruz Biotechnology) and mouse monoclonal anti-caspase-3 (sc-271759, Santa Cruz Biotechnology) at 1:500 dilution in 5\%BSA/TBS-T. After primary antibody incubation, membranes were washed 6 times with 1XTBS-T buffer at room temperature each for $5 \mathrm{~min}$ and incubated with 1:1000 diluted anti-mouse lgGk light chain binding protein (m-lgGK BP) conjugated to HRP (sc-516102, Santa Cruz Biotechnology) for $1 \mathrm{~h}$ at room temperature. Blots were visualized using Clarity Western ECL Substrate Kit (1705061, BioRad). Blots were scanned and the densities of the specific bands were quantified and normalized with calnexin as internal loading control using ChemiDoc $^{\mathrm{TM}}$ Imaging Systems (BioRad) and Lab 4.0 Software.

\section{Integrative Pixel Analysis}

For western blot images, Photoshop CS6 Software was used to analyze the relative intensity of the protein bands. The relative expressional value was normalized to ' 1 ' in untreated control cells for each protein that was analyzed, and relative protein expression fold changes of experimental groups were then calculated.

\section{Statistical Analysis}

Statistical Package for the Social Sciences (SPSS) Statistical Analysis Program was used and results were statistically analyzed according to "paired-2 tailed student's t-test". p-value under 0.05 was considered significant and p-value under 0.001 was considered very significant for all statistical analysis. Error bars in the graphs were generated using \pm s.d. values for MTS cell viability and western blotting analysis.

\section{RESULTS}

Acibenzolar-S-methyl Inhibited SH-SY5Y Neuroblastoma Cell Line Proliferation

The MTS assay showed that the cell proliferation of SH-SY5Y neuroblastoma cells was inhibited by acibenzolar-S-methyl treatment in a time and dose-dependent manner (Figures 1A-C). Inverted microscopy images were taken by Leica DMi1 Inverted Microscope for cell culture (Leica, Wetzlar, Germany). Compared with the control group, acibenzolar-S-methyl at lower concentrations (1 $\mathrm{mM}$ ) showed little inhibition effect on cell viability, while the proliferation levels of 10, 15, and 20 $\mathrm{mM}$ acibenzolar-S-methyl treatment groups were obviously decreased both at 24 and $48 \mathrm{~h}$ (data not shown for 48 hours). Since the amount of decrease is similar and adequate for half of cell growth inhibition (IC50) with both $24 \mathrm{~h}$ and $48 \mathrm{~h}$ incubation, we did not further conduct $72 \mathrm{~h}$ experiment. For SH-SY5Y cells, acibenzolar-S-methyl at the concentration of $10 \mathrm{mM}$ caused half of cell growth inhibition (IC50) at $24 \mathrm{~h}$ (Figure $1 \mathrm{~A}{ }^{* * *} \mathrm{p}<$ $0.001)$. Therefore, this concentration and this time point were used in all further experiments.

\section{Acibenzolar-S-methyl Inhibited MEK1/2 Signaling in SH- SY5Y Neuroblastoma Cell Line}

To investigate the effect of acibenzolar-S-methyl on MEK1/2 signaling, Western blotting was performed with $10 \mathrm{mM}$ acibenzolar-S-methyl -treated SH-SY5Y cells using mouse monoclonal anti- MEK1/2 (sc-81504, Santa Cruz Biotechnology) and mouse monoclonal anti-calnexin (sc-80645, Santa Cruz Biotechnology) as the internal control. Blotting results showed that MEK1/2 signaling was significantly inhibited upon acibenzolar-S-methyl treatment (Figures $2 \mathrm{~A}$ and $2 \mathrm{~B},{ }^{* * *} \mathrm{p}<0.001$ ).

\section{Acibenzolar-S-methyl Induced Caspase-3 Activity in SH- SY5Y Neuroblastoma Cell Line}

The MTS assay showed that the viability of SH-SY5Y cells was significantly decreased upon acibenzolar-S-methyl treatment which was consistent with the decrease in MEK1/2 signaling. In order to reveal whether the decrease in cell viability is associated with the activation of a major apoptotic protein, caspase-3, active-caspase 3 expression was analyzed by western blotting using mouse monoclonal anti-caspase-3 (sc-271759, Santa Cruz Biotechnology) and mouse monoclonal anti-calnexin (sc-80645, Santa Cruz Biotechnology) as the internal control. Western blotting results indicated that active-caspase 3 levels significantly increased with acibenzolar-S-methyl treatment (Figures $3 \mathrm{~A}$ and $3 \mathrm{~B},{ }^{* * *} \mathrm{p}<0.001$ ).

\section{DISCUSSION}

One of the major underlying mechanisms of diseases such as cancer, autoimmunity, and diabetes is the disruption of intracellular signaling cascades controlling cellular behavior by organizing essential cellular activities in the cell. Thus, it has become the most important focus of cancer biology to better understand the molecular mechanisms of signal transduction deficiency and its relation to cancer development (1). In this regard, numerous studies showed that SA containing low dose aspirin is protective against many cancers such as colorectal, breast, gastric, lung cancers (2-6). The increasing number of evidence indicated that SA has an apoptotic action in cancer and this action may be due to its regulatory effect on mitogenic MEK1/2 signaling cascade (9). In many cancers, inhibiting 

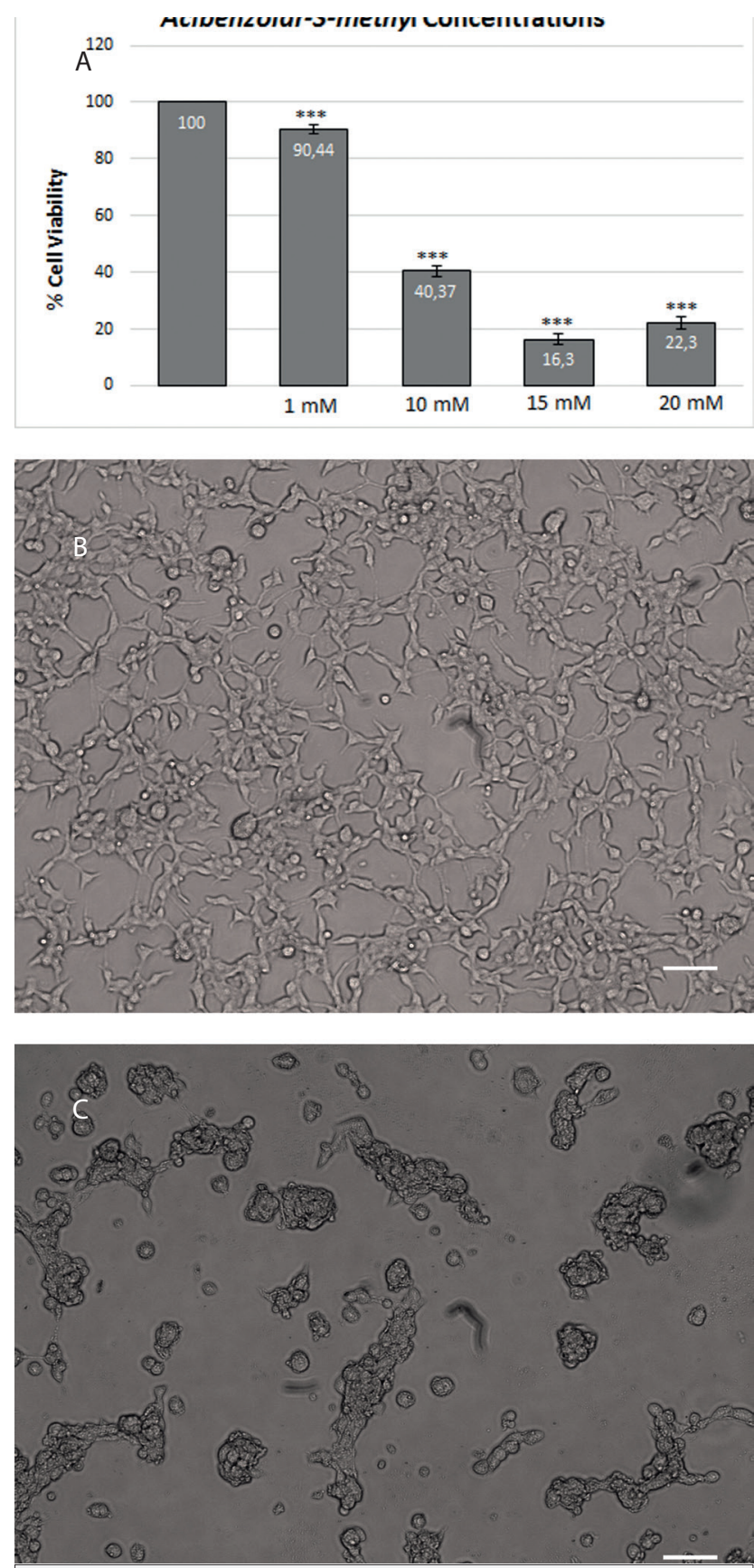

Figure 1. Inhibiting effects of acibenzolar-S-methyl on neuroblastoma cell line SH-SY5Y growth after exposure of 24h. (A) Acibenzolar-S-methyl was added to each well of a 96- well plate to yield the final concentrations of 1, 10,15 and $20 \mathrm{mM}$. Cell viability was determined by the MTS assay and absorbance was measured at $490 \mathrm{~nm}$ using a microplate reader. The graph indicates quantitative analysis of MTS results which were expressed as percentages of proliferation compared to the control. Bar represents mean values \pm SD. ${ }^{* *} p$ $<0.001$ (B) Inverted microscopy image of control SH-SY5Y cells (Magnification: 10X). The scale bar is $50 \mu \mathrm{m}$. (C) Inverted microscopy image of $10 \mathrm{mM}$ - Acibenzolar-S-methyl treated SH-SY5Y cells (Magnification: 10X). The scale bar is $50 \mu \mathrm{m}$.

\section{A \\ Control \\ Acibenzolar -S-methyl}

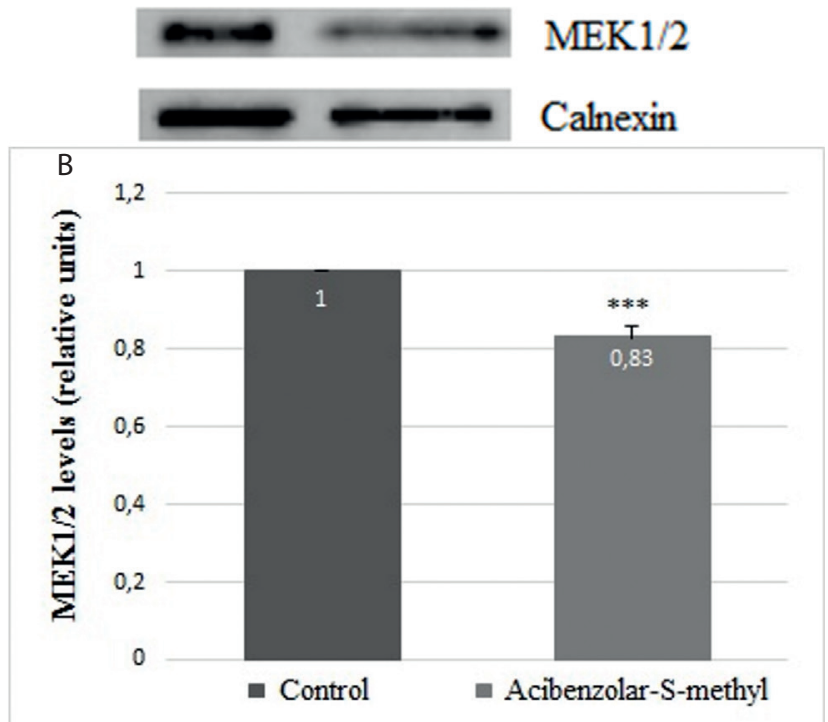

Figure 2. Inhibition effect of acibenzolar-S-methyl on MEK1/2 signaling in SH-SY5Y cells. (A) Western blotting membrane was probed with MEK1/2 antibody and calnexin antibody as internal control. (B) The graph indicates quantitative analysis of signals in Panel A. Band intensity of MEK1/2 was normalized according to calnexin. Numbers were calculated according to experimental/control ratio of MEK $1 / 2$ expression and results were represented as fold change. The bar represents mean values \pm SD. ${ }^{* *} p<0.001$

MEK1/2 signaling results in apoptotic death, while in certain types of cancers such as melanoma, inhibiting MEK1/2 signaling conversely ends up with resistance to apoptosis (10). However, a limited number of studies have been focused on the effect of this signaling pathway in neuroblastoma cancer. Moreover, there is no study about the effect of SA on MEK $1 / 2$ signaling.

In the present study, we investigated the effect of SA analog acibenzolar-S-methyl on the MEK1/2 signaling and correlatively on cell viability in neuroblastoma cells and we showed that acibenzolar-S-methyl inhibited MEK1/2 signaling and this inhibition has the potential to induce the death of SH-SY5Y neuroblastoma cells.

Our results indicated that treating SH-SY5Y cells with $10 \mathrm{mM}$ acibenzolar-S-methyl downregulated MEK1/2 signaling (Figures $2 \mathrm{~A}$ and $2 \mathrm{~B}$ ) which is consistent with the results of another study conducted in A549 human lung cancer cells demonstrating the suppressing effect of SA on MAPK signaling (9).

Furthermore, we aimed to make a preliminary interpretation about the apoptotic effect of MEK1/2 signaling inhibition and cell viability reducing effect of acibenzolar-S-methyl in SH-SY5Y neuroblastoma cells. For this reason, we analyzed a very strong apoptosis indicator, active caspase-3 protein levels by western 
blotting and we showed that there is a significant two-fold increase in active-caspase 3 levels of acibenzolar-S-methyltreated and MEK1/2 inhibited SH-SY5Y cells (Figures 3A and 3B). This result correlates with the results of the studies performed with human colorectal tumor cell lines and hepatoma cell line showing dose- and time-dependent apoptotic effect of SA $(7,8)$, and also with the results of the study performed with A549 human lung cancer cells demonstrating the suppressing and apoptotic effect of SA on MAPK signaling (9). On the other hand, our result is inconsistent with the results of the study showing that inhibiting MEK1/2 signaling contributes to resistance to apoptosis in melanoma cells (10). This contradiction implies that the molecular basis of cancer may be quite heterogeneous and thereby peculiar to each type of cancer. Thus, all these results strongly emphasize the importance of a better understanding of each cancer type-specific molecular mechanism in order to find an effective way to regulate the right targets for convenient treatment modalities. For this reason, although our active caspase- 3 analysis implies that SA may be apoptotic against certain cancers, further apoptosis studies are needed to conclude a causal association between acibenzolar-S-methyl treatment and apoptosis in SH-SY5Y cells.
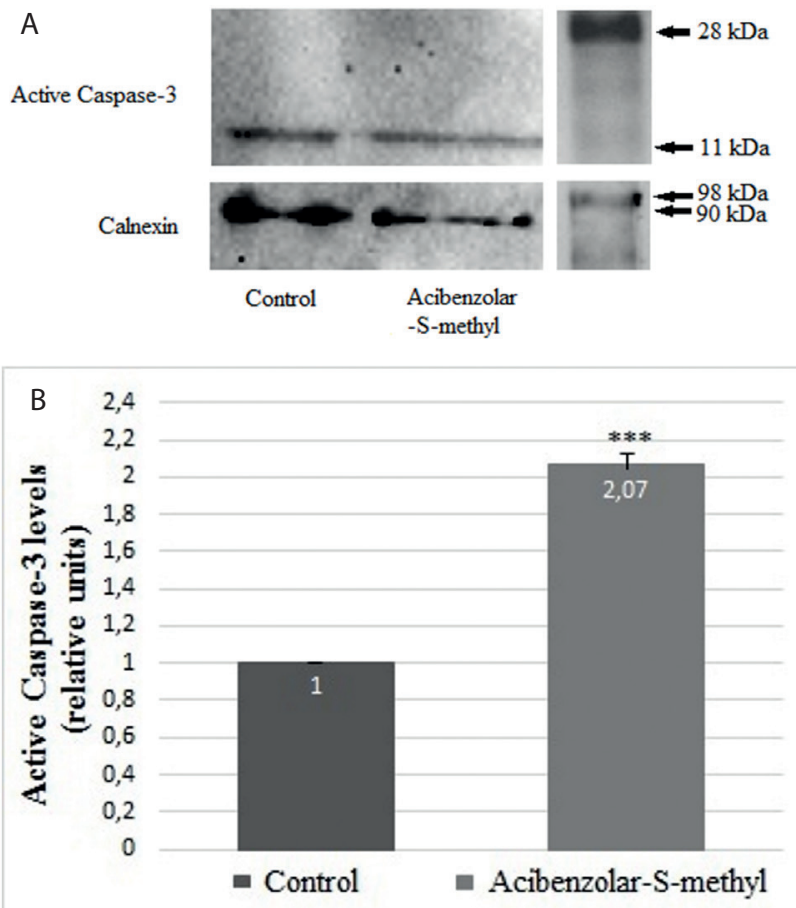

Figure 3. Activation of caspase-3 upon acibenzolar-S-methyl treatment in SH-SY5Y cells. (A) Western blotting membrane was probed with caspase-3 antibody and calnexin antibody as internal control. (B) Graph indicates quantitative analysis of signals in Panel A. Band intensity of caspase-3 was normalized according to calnexin. Numbers were calculated according to experimental/control ratio of caspase-3 expression and results were represented as fold change. The bar represents mean values \pm SD. ${ }^{* *} p<0.001$
For further studies, investigating the utility of SA or its analogs as anti-cancer agents in neuroblastoma, non-cancerous healthy cell lines may be utilized to clearly analyze and compare the effects and side effects of SA in both cancerous and non-cancerous cells. Besides, in vitro studies should be performed with neuroblastoma patient samples as well as in vivo studies with disease models to analyze its exact effectiveness in living organisms.

\section{CONCLUSION}

In this study, we showed for the first time that SA analog acibenzolar-S-methyl negatively affects MEK1/2 signaling and decreases viability of SH-SY5Y neuroblastoma cells. Since there are studies demonstrating the cancer-promoting antiapoptotic properties of SA in some cancers such as melanoma, to obtain a preliminary apoptosis data we performed active caspase-3 analysis and showed that it may be pro-apoptotic for SH-SY5Y neuroblastoma cells which needs further exploration. In this regard, considering the heterogeneous and idiosyncratic nature of each cancer type, this study has the potential to enable the selection of appropriate therapeutic targets and the development of effective diagnosis and treatment strategies for many cancers, particularly for neuroblastoma cancer.

Peer-review: Externally peer-reviewed.

Author Contributions: Conception/Design of study: A.Y.; Data Acquisition: A.Y.; Data Analysis/Interpretation: A.Y.; Drafting Manuscript: A.Y.; Critical Revision of Manuscript: A.Y.; Final Approval and Accountability: A.Y.

Conflict of Interest: The author declare that they have no conflicts of interest.

Financial Disclosure: This study was supported by grant to Ayşegül Yıldız from Mugla Sitki Kocman University Scientific Research Project Office, Research and Development Projects (Project No: 17/023).

Acknowledgements: I would like to thank Assoc.Prof.Dr. Esin Sakallı ÇETiN from Muğla Sıtkı Koçman University, Faculty of Medicine, Medical Biology Department and Prof. Dr. Arzu KARABAY KORKMAZ from Istanbul Technical University, Faculty of Science and Letters, Molecular Biology and Genetics Department for allowing me to use their laboratory infrastructure. I also would like to thank Prof. Dr. Ömür BAYSAL from Muğla Sıtkı Koçman University, Faculty of Science, Molecular Biology and Genetics Department for his generous gift of acibenzolar-S-methyl (Syngenta).

\section{REFERENCES}

1. Çoban ZD, Güran Ş. The role of signal transducing mechanisms in cancer diagnosis and treatment. Cumhuriyet Medical Journal 2013; 35: 302-10.

2. Brasky TM, Bonner MR, Moysich KB, Ambrosone CB, Nie J, Tao MH. Non steroidal anti-inflammatory drug use (NSAID) and breast cancer risk in the western New York Exposures and breast cancer (WEB) study. Cancer Causes Control 2010; 9: 1503-12. 
3. Rothwell PM, Wilson M, Price JF, Belch JF, Meade TW, Mehta Z. Effect of Daily aspirin on risk of cancer metastasis: a study of incident cancers during randomised controlled trials. Lancet 2012; 379:1591-601.

4. Elwood PC, Morgan G, Pickering JE, Galante J,Weightman AL, Morris D, Kelson M, Dolwani S. Aspirin in the treatment of cancer: reductions in metastatic spread and in mortality: a systematic review and meta-analyses of published studies. PLoS One. 2016; 11(4): e0152402. doi:10.1371/ journal.pone.0152402

5. Klessig DF. Newly identified targets of aspirin and its primary metabolite, salicylic acid. DNA Cell Biol 2016; 35(4):163-6. doi:10. 1089/dna.2016.3260

6. Giampieri R, Restivo A, Pusceddu V, Del Prete M, Maccaroni E, Bittoni A, Faloppi L, Andrikou K, Bianconi M, Cabras F, Berardi R, Zorcolo L, Scintu F, Cascinu S, Scartozzi M. The role of aspirin as antitumoral agent for heavily pretreated patients with metastatic colorectal cancer receiving capecitabine monotherapy. Clin Colorectal Cancer 2016;16(1):38-43. doi:10.1016/j.clcc.2016.07.011

7. Liu Y, Wang Y, Li L, Hu Y, Ge S, Li K, Wang S. The apoptotic inducible effects of salicylic asid on hepatoma cell line: relationship with nitric oxide signaling. J Cell Commun Signal 2017; 11: 245-53.

8. Elder DJ, Hague A, Hicks DJ, Paraskeva C. Differential Growth Inhibition by the aspirin metabolite salicylate in human colorectal tumor cell lines: Enhanced apoptosis in carcinoma and in vitrotransformed adenoma relative to adenoma cell lines. Cancer Research 1996; 56: 2273-76.
9. Pan MR, Chang HC, Hung WC. Non-steroidal anti-inflammatory drugs suppress the ERK signaling pathway via block of Ras/c-Raf interaction and activation of MAP kinase phosphatases. Cell Signal 2008; 20(6):1134-41.

10. Haydn JM, Hufnagel A, Grimm J, Maurus K, Schartl M, Meierjohann $\mathrm{S}$. The MAPK pathway as an apoptosis enhancer in melanoma. Oncotarget 2014; 5(13): 5040-53.

11. Dhillon AS, Hagan $S$, Rath $O$, Kolch W. MAP kinase signalling pathways in cancer. Oncogene 2007;26(22):3279-90.

12. Mattingly RR, Milstein ML, Mirkin BL. Down-regulation of growth factor-stimulated MAP kinase signaling in cytotoxic drug-resistant human neuroblastoma cells. Cell Signal 2001; 13(7):499-505.

13. Maris JM. Recent advances in neuroblastoma. N Engl J Med 2010; 362(23):2002.

14. Lubanska D, Market B, de Calvalho A., Mikkelson T, Fidalgo da Silva E, Porter LA. The atypical cell cycle regulator Spy1 suppresses differentiation of the neuroblastoma stem cell population. Oncoscience $2014 ; 25$ : 64-7. 